\title{
NADPH as a potential intrinsic probe for tumour margin estimation
}

\author{
Hazel L. Stewart*a, Ted R. Hupp ${ }^{\mathrm{b}}$, David J. S. Birch ${ }^{\mathrm{a}}$ \\ ${ }^{a}$ Photophysics Group, Department of Physics, University of Strathclyde, 107 Rottenrow East, \\ Glasgow, G4 0NG; ${ }^{b}$ Edinburgh Cancer Research UK Centre, University of Edinburgh, Crewe Road \\ South, Edinburgh, EH4 2XR
}

\begin{abstract}
The fluorescent properties of the reduced coenzyme NADH and its phosphorylated derivative (NADPH) have been explored in order to assess its potential as an intrinsic probe for cancer surgery. NADPH production is increased in cancer cells to quench reactive oxygen species and meet higher demands for biosynthesis, and has attractive fluorescent properties such as emission towards the visible part of the spectrum and a relatively long fluorescence lifetime upon binding to enzymes $(\sim 1-6.5 \mathrm{~ns})$ that help discriminate against other endogenous species. Different environmental effects on $\mathrm{NAD}(\mathrm{P}) \mathrm{H}$ fluorescence are reported here, including an increase in lifetime upon oxygen removal, an ability to retain its fluorescent properties in a silica phantom and its fluorescence lifetime also being distinguishable in a cell environment. In addition to this, the development of a miniaturised liquid light guide filter-based time-correlated single photon counting fluorescence lifetime system is reported as a step towards time-resolved visual imaging in cancer surgery. This system has been demonstrated as being capable of measuring $\mathrm{NAD}(\mathrm{P}) \mathrm{H}$ fluorescence lifetimes in both simple solvent and cell environments.
\end{abstract}

Keywords: fluorescence lifetime, NADPH, fluorescence spectroscopy, tumour margin estimation, liquid light guide, intrinsic probe

\section{INTRODUCTION}

Cancer is one of the leading causes of death worldwide, with approximately 14 million new cases being diagnosed in 2012 alone $^{1}$. This number of new cases is expected to rise by approximately $70 \%$ over the next 20 years ${ }^{1}$, and so improving treatment and diagnosis options for cancer remains of high importance. One of the primary treatment options for tumour removal is surgery, where in the UK in $201446 \%$ of patients diagnosed with cancer underwent surgery to remove the tumour as part of the treatment plan, compared to $27 \%$ undergoing radiation therapy and $28 \%$ having chemotherapy ${ }^{2}$. During cancer surgery, surgeons most commonly rely on visual inspection and palpation in order to determine how much tissue surrounding the cancerous cells should be removed. While these methods can be used for determining bulk tumour, they are not sensitive enough to identify cancer at the cellular level ${ }^{3}$, which can lead to cancerous cells remaining in the body and further surgery for the patient. There are methods available for determining surgical margins of certain cancers intraoperatively, examples of which include intraoperative MRI (iMRI) or having a pathologist present to assess the tissue, however such methods have their own drawbacks such as being time consuming, requiring specialist personnel, or requiring expensive surgical suites in the case of iMRI ${ }^{4}$. Surgeons and medical experts have highlighted the limited capabilities in real-time margin estimation at the moment, where it is clear a device that can significantly reduce repeat surgery rate, take under 20 minutes to display results, have a sensing depth of $1-2 \mathrm{~mm}$ and not require specialist personnel is needed in cancer surgery ${ }^{5}$.

*h.stewart@strath.ac.uk; phone +44 (0)141 5483059 
Optical imaging techniques have been established as powerful methods in intraoperative cancer surgery due to the relatively low cost of devices, intuitive operation and ease of image acquisition ${ }^{4}$, where fluorescence-based optical imaging techniques such as fluorescence-guided surgery have obtained a growing amount of interest in particular. Nearinfrared methods are most often used, where for example the fluorescent probe indocyanine green (ICG) remains the most commonly studied and utilised fluorophore for applications in fluorescence-guided surgery. ICG has demonstrated potential in various oncological applications through combination with endoscopic-based techniques such as laparoscopy ${ }^{6}$, as well as demonstrating its potential use in breast cancer margin estimation ${ }^{7,8}$. Despite success with ICG, near-infrared imaging techniques have not readily entered into clinical use due to the lack of appropriate contrast agents that have been FDA approved with current imaging technologies ${ }^{9}$. The main problems with developing a contrast agent include the significant amount of time and money required to design a probe and bring it to market for human use. However endogenous fluorophores are a potential alternative to extrinsic probes, as they offer the major advantage of no external agent being required in order to monitor its fluorescent properties, and therefore have significantly less regulatory requirements that need to be met for their use in clinical applications ${ }^{4}$.

The reduced form of the coenzyme nicotinamide adenine dinucleotide (NADH) and its phosphorylated derivative (NADPH) are the endogenous fluorophores explored in this work for their potential use as a probe in tumour margin estimation, where their structures are shown in Figure 1.

a)

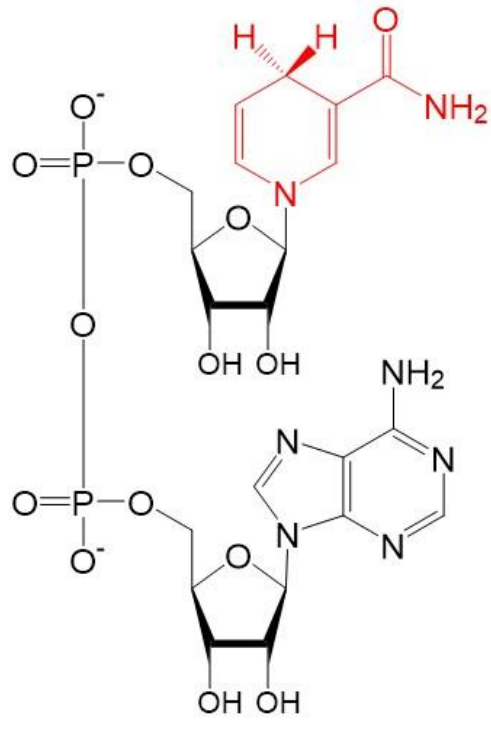

b)

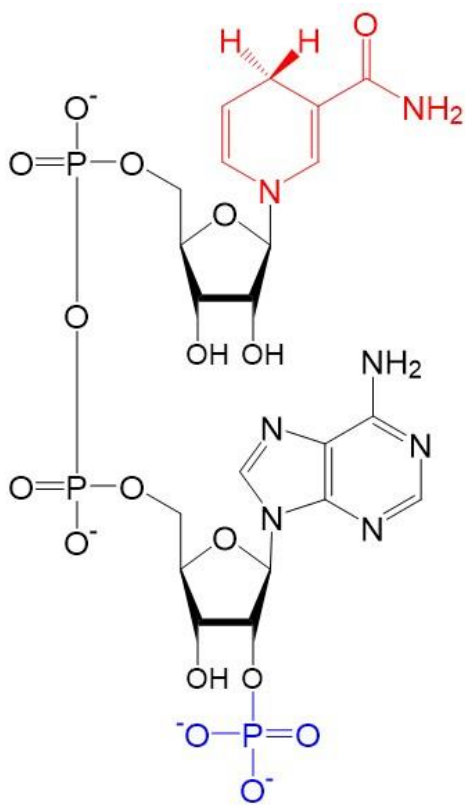

Figure 1. Chemical structures of a) NADH and b) NADPH, where the redox-active group responsible for fluorescence is shown in red, and the additional phosphate group of NADPH in blue.

NADH and NADPH (referred to together as NAD(P)H) have very similar structures but differing roles in the body. $\mathrm{NADH}$ is an electron carrier produced in cell respiration, where it carries electrons to the electron transport chain to be used in oxidative phosphorylation in cell respiration for the production of $\mathrm{ATP}^{10}$. NADPH is instead produced in the oxidative phase of the pentose phosphate pathway, where it is used in macromolecular biosynthesis and for the quenching of reactive oxygen species ${ }^{11}$. It has been shown that the pentose phosphate pathway is upregulated in cancer cells for an increase in NADPH production in particular, in order to meet the increased demand for biosynthesis in these cells as well as to quench the elevated levels of reactive oxygen species also present ${ }^{12}$. Obtaining a better understanding of the redox state of cells using $\mathrm{NAD}(\mathrm{P}) \mathrm{H}$ fluorescence has also been of interest in recent years ${ }^{13-15}$ due to the involvement of both of these coenzymes in several reaction pathways of cancer cells. 
While NADH and NADPH have different roles in the body, both fluoresce in their reduced states and have identical fluorescent properties due to their similar structures. It is the nicotinamide ring responsible for fluorescence in $\mathrm{NAD}(\mathrm{P}) \mathrm{H}$ (highlighted in red in Figure 1), which remains unaffected by the additional phosphate group in NADPH as this is at a remote site of the molecule ${ }^{16}$. As well as being an intrinsic fluorophore, NAD(P)H also offers the advantage of having fluorescence emission towards the visible part of the spectrum $\left(\lambda_{\mathrm{em}}\right.$ (max.) $\left.\sim 460 \mathrm{~nm}\right)$ compared to other intrinsic fluorophores which often fluoresce at lower wavelengths. A combination of these advantageous properties alongside the fluorescence lifetime properties of $\mathrm{NAD}(\mathrm{P}) \mathrm{H}$, which includes a 2-exponential decay with increased lifetime components upon binding to enzymes (increasing from $\sim 0.4-0.8$ to $\sim 1-6.5 \mathrm{~ns})^{17}$, should help distinguish it from other fluorescing components within cells.

The work presented here focuses on the fluorescence lifetime properties of NAD $(\mathrm{P}) \mathrm{H}$ in particular. Many procedures and instruments used in fluorescence-guided surgery use fluorescence intensity as a means of locating tissue of interest, however time-resolved techniques such as fluorescence lifetime measurements can provide additional information about a system and offer many advantages over fluorescence intensity. These advantages include the ability to discriminate against scattered light and other endogenous fluorophores, as well as being independent from emission wavelength and photobleaching, both of which can often affect fluorescence intensity measurements ${ }^{18}$. Therefore to address the needs of both an improved probe and improved instrument for margin estimation, this work ties together studies on the fluorescence lifetime properties of NADPH that make it a potentially suitable probe as well as the demonstration of fluorescence lifetime measurements using a liquid light guide-based system, for their potential use in real-time tumour margin estimation.

\section{MATERIALS \& METHODS}

\subsection{Sample preparation}

To measure the fluorescence lifetime of NADH before and after oxygen removal, stock solutions of NADH were prepared at concentrations of $80 \mu \mathrm{M}$ by dissolving $1.70 \mathrm{mg}$ of powdered reduced disodium salt hydrate $\beta$-nicotinamide adenine dinucleotide (obtained from Sigma Aldrich) in $30 \mathrm{~mL}$ of the solvents water, Trizma, PBS and ethylene glycol. Final samples were prepared at $40 \mu \mathrm{M}$ concentrations by adding $1.5 \mathrm{~mL}$ of each stock solution to separate cuvettes with a further $1.5 \mathrm{~mL}$ of solvent added to each cuvette. Cuvettes were then sealed with a rubber stopper and further secured with parafilm. To remove the oxygen, argon gas was bubbled through the solutions for $\sim 5$ minutes per sample using BD Microlance 3 needles, where the samples were resealed with more parafilm for fluorescence lifetime measurements to be conducted immediately.

For measurements of NADH in the silica hydrogel environment, a stock solution of $4.25 \mathrm{mg}$ NADH in $30 \mathrm{~mL}$ water to give a $200 \mu \mathrm{M}$ concentration was first prepared. The hydrogel itself was then prepared from methods derived from previous works ${ }^{19}$, where $10 \mathrm{~mL}$ of sodium silicate $(4 \% \mathrm{v} / \mathrm{v})$ was mixed for $\sim 5$ minutes with $1.5 \mathrm{~mL}$ of sulphuric acid $(2 \% \mathrm{v} / \mathrm{v}) .0 .5 \mathrm{~mL}$ of the $\mathrm{NADH}$ stock solution was then added to a cuvette with $2 \mathrm{~mL}$ of hydrogel solution to produce a $40 \mu \mathrm{M}$ sample of NADH in hydrogel. The sample was sealed with parafilm and mixed by hand for $\sim 1$ minute, where gelation occurred within 15 minutes of mixing. Silica hydrogels are more often manufactured on an industrial scale, where monosilicic acid condensates to form siloxane monomeric structures, which aggregate to form nanoparticles that grow in size. The network of particles produced will eventually span the vessel and gel at a time $t_{g}$ known as the gelation time $^{20}$. The polymerisation of hydrogels occurs through hydrolysis and condensation reactions given by:

$$
\begin{array}{ll}
\mathrm{Na}_{2} \mathrm{O} \cdot x \mathrm{SiO}_{2} \cdot y \mathrm{H}_{2} \mathrm{O}+\mathrm{H}_{2} \mathrm{SO}_{4} \rightarrow \mathrm{Si}(\mathrm{OH})_{4}+\mathrm{Na}_{2} \mathrm{SO}_{4} & \text { (Hydrolysis) } \\
\mathrm{Si}(\mathrm{OH})_{4}+\mathrm{Si}(\mathrm{OH})_{4} \rightarrow 2 \mathrm{SiO}_{2}+4 \mathrm{H}_{2} \mathrm{O} & \text { (Condensation) }
\end{array}
$$


Silica alcogels were also used in measurements of NADH in a sol-gel environment. Silica alcogels are of a higher purity and have better defined structures compared to hydrogels, where tetraethylorthosilicate (TEOS) is used in the preparation instead leading to a hydrolysis reaction of:

$$
\mathrm{Si}\left(\mathrm{OC}_{2} \mathrm{H}_{5}\right)_{4}+4 \mathrm{H}_{2} \mathrm{O} \rightarrow \mathrm{Si}(\mathrm{OH})_{4}+4 \mathrm{C}_{2} \mathrm{H}_{5} \mathrm{OH} \quad \text { (Hydrolysis) }
$$

which then leads to the condensation reactions shown in equation 2. For NADH silica alcogel samples, stock solutions of $80 \mu \mathrm{M}$ NADH in PBS were first prepared as described previously. The alcogel itself was prepared by mixing $8 \mathrm{~mL}$ of TEOS with $3 \mathrm{~mL}$ of water and $0.2 \mathrm{~mL}$ of $0.01 \mathrm{M}$ hydrochloric acid for 2 hours $^{21}$. Once mixed, $1.5 \mathrm{~mL}$ of alcogel was added to $1.5 \mathrm{~mL}$ of NADH in PBS in a cuvette, which was sealed and then mixed for a further minute, where $t_{g} \sim 5$ minutes.

The fluorescent dye Rhodamine 6G (Sigma Aldrich) and the xanthene based probe JA120 (supplied through collaboration with the University of Siegen) used in measurements on the liquid light guide-based system were prepared by dissolving the powder form of each probe in methanol (Rhodamine 6G) and water (JA120). These dyes were chosen for measurements on the light guide system due to their short lifetimes $\left(\sim 4 \mathrm{~ns}\right.$ for Rhodamine $6 \mathrm{G}^{22}, \sim 1.8 \mathrm{~ns}$ for $\mathrm{JA} 120^{23}$ ), which are close to that of $\mathrm{NAD}(\mathrm{P}) \mathrm{H}$ in both bound and unbound form. The structures of these fluorophores are shown in Figure 2.

a)

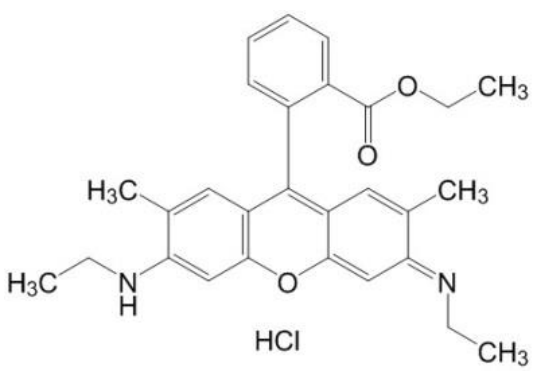

b)

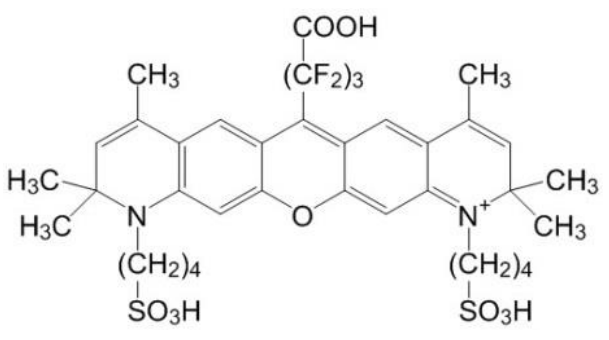

Figure 2. Chemical structures of a) Rhodamine $6 \mathrm{G}$ and b) xanthene type probe JA120.

The volume of stock solution of each dye that was required to give an absorbance of $\sim 0.1$ in $3 \mathrm{~mL}$ of solvent was determined, where this absorbance is strong enough to generate a good fluorescence signal but will not allow for problems with dye-dye interactions ${ }^{24}$. The determined volume was then added to cuvettes with $3 \mathrm{~mL}$ of water and sealed to perform lifetime measurements.

For cell measurements, PNT2 cells (normal prostate epithelium, human) were first cultured in Roswell Park Memorial Institute medium (RPMI) under normal conditions, where they were incubated at $37^{\circ} \mathrm{C}$ with $5 \% \mathrm{CO}_{2}$. Cells were placed in a sterile tube, where the volume of cells required to give a concentration of $1 \times 10^{6}$ cells $/ \mathrm{mL}$ in $2 \mathrm{~mL}$ of solvent was calculated. This determined volume was then added to $2 \mathrm{~mL}$ of PBS in a cuvette and then sealed with parafilm ready to perform fluorescence lifetime measurements.

\subsection{Fluorescence lifetime measurements}

Measurements of NADH in the oxygen purged and silica sol-gel environments were made using time correlated single photon counting (TCSPC) on a Horiba Scientific Deltaflex system as shown in Figure 3a. The instrument incorporates a monochromator on the emission arm as a means of wavelength selection, where fluorescence photons are detected using a TBX photon detection module. The polariser on the excitation arm is set to a vertical position, where the polariser on the emission arm is also set to a vertical position to measure the instrument response function (IRF), and is then switched to the magic angle $\left(54.7^{\circ}\right)$ for fluorescence lifetime measurements. Samples were measured until 10,000 counts in the peak of the fluorescence lifetime decay were collected. 


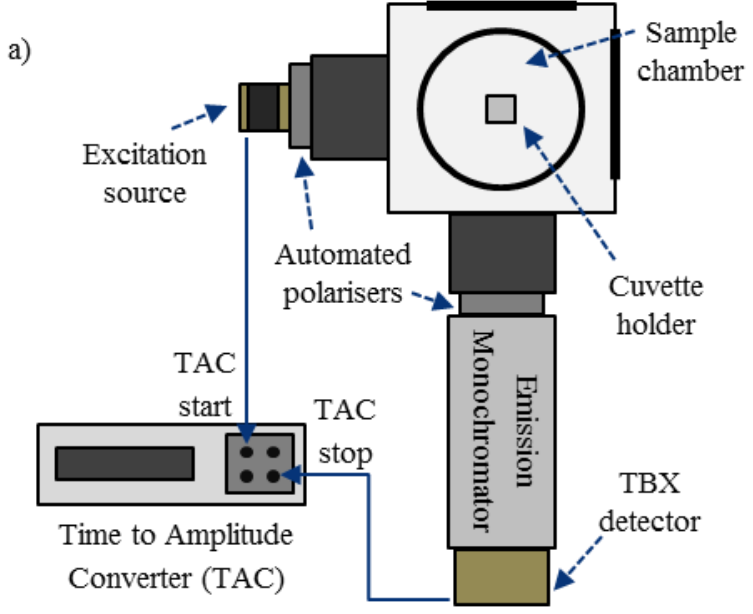

b)

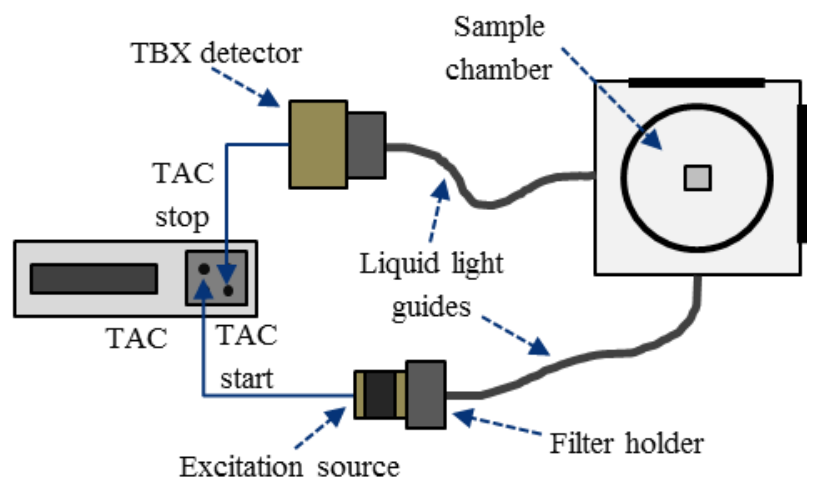

Figure 3. Schematic for TCSPC setup of a) Horiba Scientific Deltaflex and b) modified Horiba TemPro system incorporating liquid light guides as a means of excitation and collection of fluorescence.

The liquid light guide-based fluorescence lifetime system was developed from a Horiba TemPro system, where the original arms of the instrument have been removed and instead have mounts for the liquid light guides to go in and out the sample chamber as shown in Figure $3 \mathrm{~b}$. The liquid light guides are used as a means of sample excitation and collection of the fluorescence signal. Liquid light guides have advantages over conventional silica fibre bundles which are more often used in surgical applications, such as having an ability to transmit more light due to the large cross section, an increased flexibility and a higher numerical aperture for a larger acceptance angle of light ${ }^{25}$. Previous work in the Photophysics group has demonstrated the capabilities of liquid light guides in miniaturised fluorescence lifetime systems, where the use of $50 \mathrm{~cm}$ light guides of a $0.3 \mathrm{~cm}$ diameter have been used in a multiplexed fluorometer ${ }^{26,27}$, where here we have incorporated $1 \mathrm{~m}$ light guides with a $0.3 \mathrm{~cm}$ diameter for use in single lifetime measurements. The couplings of the light guides to the detector and excitation source have space to hold neutral density and/or longpass filters as required, where these couplings were made as light tight as possible to minimise interference from background light. The fluorescence lifetime decays of NADH presented in this work were measured using a NanoLED excitation source of $339 \mathrm{~nm}$ on both systems, with emission wavelengths of $460 \mathrm{~nm}$ and $450 \mathrm{~nm}$ on the DeltaFlex and TemPro systems respectively. Rhodamine $6 \mathrm{G}$ samples were excited at $474 \mathrm{~nm}$ with an emission wavelength of $560 \mathrm{~nm}$, and JA120 samples were excited at $638 \mathrm{~nm}$ with emission recorded at $685 \mathrm{~nm}$. For cell measurements, the original setup of the Horiba TemPro system and the adapted liquid light guide system were used for comparison, where the original TemPro system is the same as the DeltaFlex system in Figure 3a, but instead uses longpass filters rather than a monochromator for emission wavelength selection. Data analysis for all measurements was performed using the Horiba Scientific DAS6 software package using non-linear least squares (NLLS) reconvolution analysis.

\section{RESULTS AND DISCUSSION}

\subsection{Effect of oxygen quenching on NADH fluorescence}

The effect of oxygen on the fluorescence lifetime of NADH in various solvents has been investigated. Cancer cells are known to have depleted levels of oxygen ${ }^{28}$, so a difference in the fluorescence lifetime of NADH between the two environments with and without oxygen may provide a potential method for determining between cancerous and healthy cells. Four different solvents - water, PBS, Trizma and ethylene glycol - were used. No difference in lifetime was observed for NADH in Trizma and PBS; however changes in the lifetime in water and ethylene glycol are shown in Figure 4 and Table 1. 


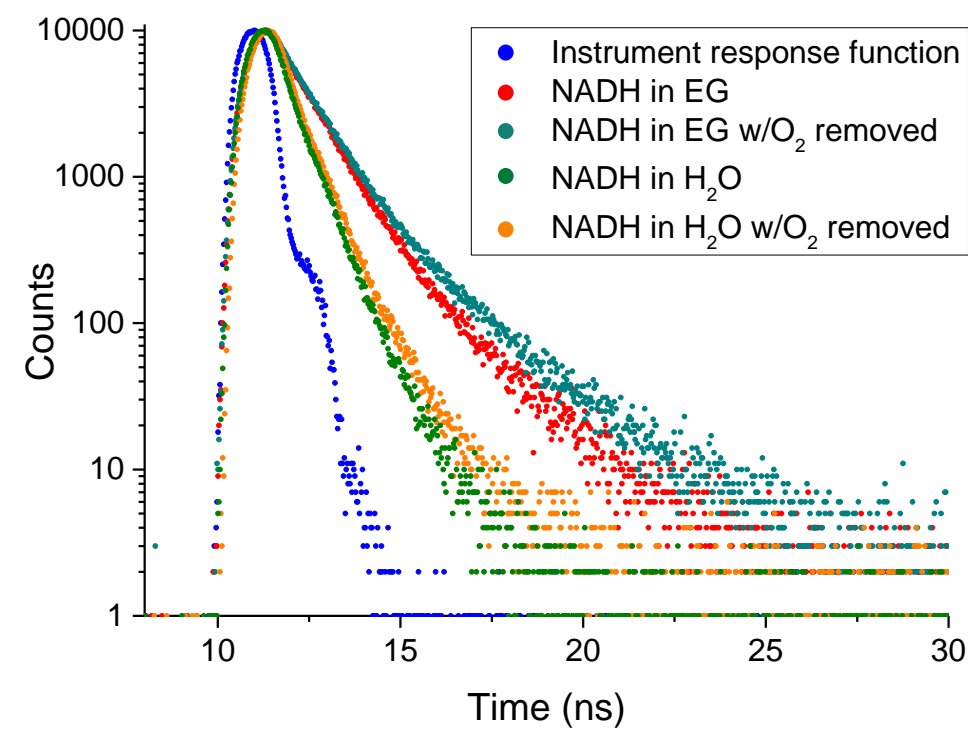

Figure 4. Fluorescence lifetime decays of NADH in water and ethylene glycol (EG) before and after oxygen is removed from the sample.

Table 1. Fluorescence lifetime components obtained from the fluorescence lifetime decays shown in Figure 4 with corresponding contribution of each component to the fluorescence decay.

\begin{tabular}{|c|c|c|c|c|c|}
\hline NADH Sample & $\boldsymbol{\tau}_{\mathbf{1}}(\mathbf{n s})$ & $\mathbf{B}_{\mathbf{1}}(\boldsymbol{\%})$ & $\boldsymbol{\tau}_{\mathbf{2}}(\mathbf{n s})$ & $\mathbf{B}_{\mathbf{2}}(\boldsymbol{\%})$ & $\chi^{\mathbf{2}}$ \\
\hline $\mathrm{H}_{2} \mathrm{O}$ & $0.26 \pm 0.03$ & 51.06 & $0.63 \pm 0.01$ & 48.94 & 1.10 \\
\hline $\mathrm{H}_{2} \mathrm{O} \mathrm{w} / \mathrm{O}_{2}$ removed & $0.35 \pm 0.02$ & 70.55 & $0.86 \pm 0.02$ & 29.45 & 1.11 \\
\hline Ethylene glycol & $0.77 \pm 0.01$ & 86.14 & $1.72 \pm 0.07$ & 13.86 & 1.20 \\
\hline Ethylene glycol w/O $\mathrm{O}_{2}$ removed & $0.77 \pm 0.01$ & 88.41 & $1.94 \pm 0.08$ & 11.59 & 1.10 \\
\hline
\end{tabular}

The effect of oxygen can be seen looking at the fluorescence lifetime decays in Figure 4 alone, where there is a clear increase in each decay when oxygen has been removed from the samples. Further to this, Table 1 shows that there is an increase in both $\tau_{1}$ and $\tau_{2}$ when NADH is in water and an increase in $\tau_{2}$ for NADH in ethylene glycol. There is also a significant increase in the contribution of $\tau_{1}$ when NADH is in water as well. Changes in lifetime would be expected due to the efficiency of oxygen as a quencher, where the differing viscosities of water and ethylene glycol is likely to be a contributing factor to the bigger change being observed in water. Using the Stokes-Einstein equation for diffusing molecules in a viscous fluid:

$$
D=\frac{k T}{6 \pi \mu r}
$$

where $k$ is the Boltzmann constant, $T$ is temperature, $\mu$ is the solvent viscosity and $r$ is the radius of the diffusing particle, we can see that an increased viscosity such as that of ethylene glycol would result in a lower rate of diffusion for the molecules in this solvent. This means that the efficiency of oxygen as a quencher is reduced in the ethylene glycol environment. The increase in fluorescence lifetime of NADH in water is important to note here. As water is the most abundant molecule in cells ${ }^{29}$, this change in lifetime could indicate a potential method of determining the environment that NADPH is in, where a longer lifetime could indicate an oxygen deprived i.e. tumour environment.

\subsection{NADH fluorescence in a silica sol-gel environment}

Silica sol-gels are extremely effective scatterers, where here both hydrogels and alcogels have been used as rough human tissue mimics, as human tissue is also known to be highly scattering. Many different types of phantom are available in 
solid and liquid form, where both can be adapted and tuned to match the absorption and scattering properties of the different types of tissue in the body. Phantoms have been developed by various groups for their specific use with different techniques such as photodynamic therapy ${ }^{30}$, fluorescence spectroscopy ${ }^{31}$ and ultrasound ${ }^{32}$, where here we have chosen to use silica sol-gels for their ease of production and highly scattering properties. The fluorescence decays obtained for NADH in both the silica hydrogel and alcogel are shown in Figure 5, where the lifetime parameters obtained for each measurement are shown in Table 2.

a)

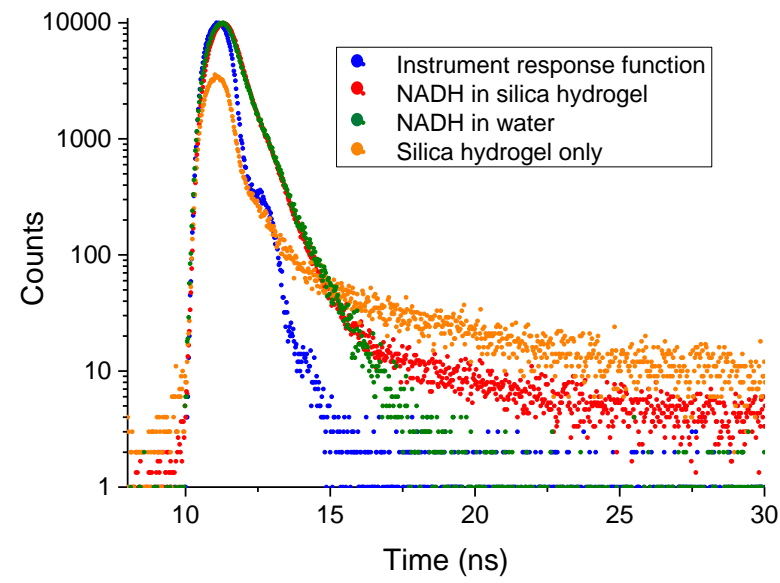

b)

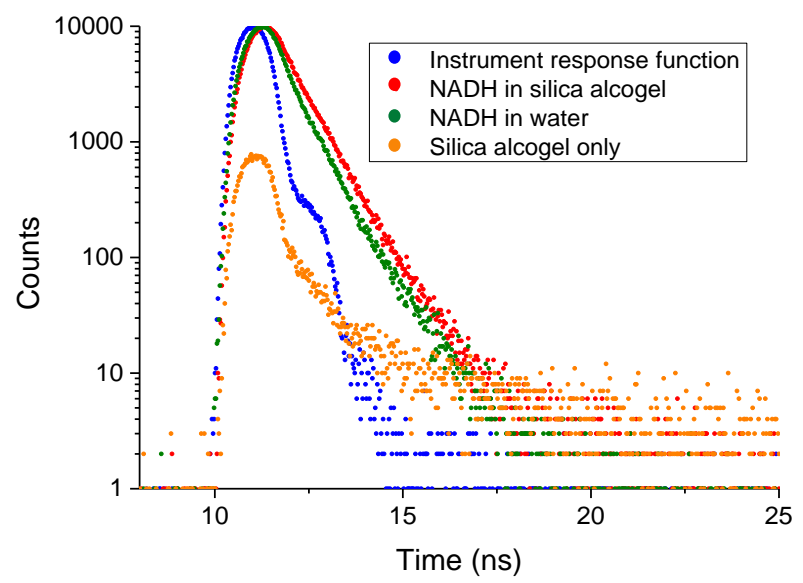

Figure 5. Fluorescence lifetime decays of NADH in a) a silica hydrogel and b) silica alcogel environment, where the decay of NADH in water and the decay of the silica hydrogel and alcogel alone obtained in the same time frame are shown for comparison.

Table 2. Fluorescence lifetime components obtained from the fluorescence lifetime decays shown in Figure 5 with corresponding contribution of each component to the fluorescence decay.

\begin{tabular}{|c|c|c|c|c|c|c|c|}
\hline NADH Sample & $\boldsymbol{\tau}_{\mathbf{1}}(\mathbf{n s})$ & $\mathbf{B}_{\mathbf{1}}(\boldsymbol{\%})$ & $\boldsymbol{\tau}_{\mathbf{2}}(\mathbf{n s})$ & $\mathbf{B}_{\mathbf{2}}(\boldsymbol{\%})$ & $\boldsymbol{\tau}_{\mathbf{3}}(\mathbf{n s})$ & $\mathbf{B}_{\mathbf{3}}(\boldsymbol{\%})$ & $\chi^{\mathbf{2}}$ \\
\hline NADH in silica hydrogel & $0.61 \pm 0.04$ & 46.58 & $3.88 \pm 1.13$ & 1.22 & $0.26 \pm 0.02$ & 52.20 & 1.19 \\
\hline Silica hydrogel only & $1.31 \pm 0.13$ & 10.23 & $8.88 \pm 0.55$ & 8.33 & $0.11 \pm 0.01$ & 81.44 & 1.19 \\
\hline NADH in silica alcogel & $0.43 \pm 0.03$ & 65.71 & $0.94 \pm 0.03$ & 34.29 & - & - & 1.17 \\
\hline Silica alcogel only & $1.08 \pm 0.14$ & 9.32 & $6.73 \pm 0.41$ & 7.48 & $0.09 \pm 0.01$ & 83.20 & 1.17 \\
\hline NADH in water & $0.35 \pm 0.03$ & 70.29 & $0.85 \pm 0.03$ & 29.71 & - & - & 1.14 \\
\hline
\end{tabular}

Figure 5a shows that the fluorescence lifetime decay of the silica hydrogel is very close to the decay of NADH in water alone, where water is being used here as a simple solvent environment for comparison. A longer component is also observed towards the end of the decay which is a contribution from the hydrogel itself, which can be determined both by comparison with the fluorescence lifetime decay of the silica hydrogel alone as well as the components shown in Table 2 , where a third exponential component was required to describe the data.

The fluorescence lifetime decay for NADH in the silica alcogel in Figure 5b again bears great similarity to the decay of $\mathrm{NADH}$ in water, where there is almost no contribution from the sol-gel itself in this case. The fluorescence decay of the silica alcogel itself has a smaller number of counts in the peak of decay compared to that of the hydrogel obtained in the same length of time, suggesting that there is less fluorescence emission from the alcogel at $\lambda_{\text {em }}=460 \mathrm{~nm}$. This means it is less likely to interfere with the fluorescence decay of NADH, which can be seen from Table 2 as only two exponential components were required to describe the decay, both of which were close to what was observed for NADH in water alone. This smaller contribution from the alcogel would be expected due to their higher purity compared to hydrogels. 


\subsection{Fluorescence lifetime measurements on the liquid light guide system}

Once the liquid light guide system was configured, fluorescence lifetime measurements of the dyes Rhodamine 6G and JA120 were performed on the system and then compared with measurements of these dyes on the full fluorescence lifetime system that incorporates an emission monochromator. A comparison of the fluorescent lifetimes obtained for each of these samples on both systems is shown in Table 3, which shows that the parameters obtained are in good agreement with each other and that therefore the liquid light guide system operates as well as the monochromator system.

Table 3. Fluorescence lifetimes obtained for Rhodamine 6G and JA120 on the monochromator system and the liquid light guide system.

\begin{tabular}{|c|c|c|c|}
\hline Sample & Lifetime System Setup & $\boldsymbol{\tau}_{\mathbf{1}}(\mathbf{n s})$ & $\chi^{2}$ \\
\hline \multirow{2}{*}{ Rhodamine 6G } & Monochromator & $4.06 \pm 0.01$ & 1.19 \\
\cline { 2 - 4 } & Liquid light guide & $4.01 \pm 0.02$ & 1.18 \\
\hline \multirow{2}{*}{ JA120 } & Monochromator & $1.75 \pm 0.01$ & 1.09 \\
\cline { 2 - 4 } & Liquid light guide & $1.79 \pm 0.01$ & 1.13 \\
\hline
\end{tabular}

Fluorescent lifetime measurements of NADH in the simple solvent environments were also performed on the liquid light guide system, where the fluorescent lifetime decays of NADH in Trizma measured on both systems are shown in Figure 6.

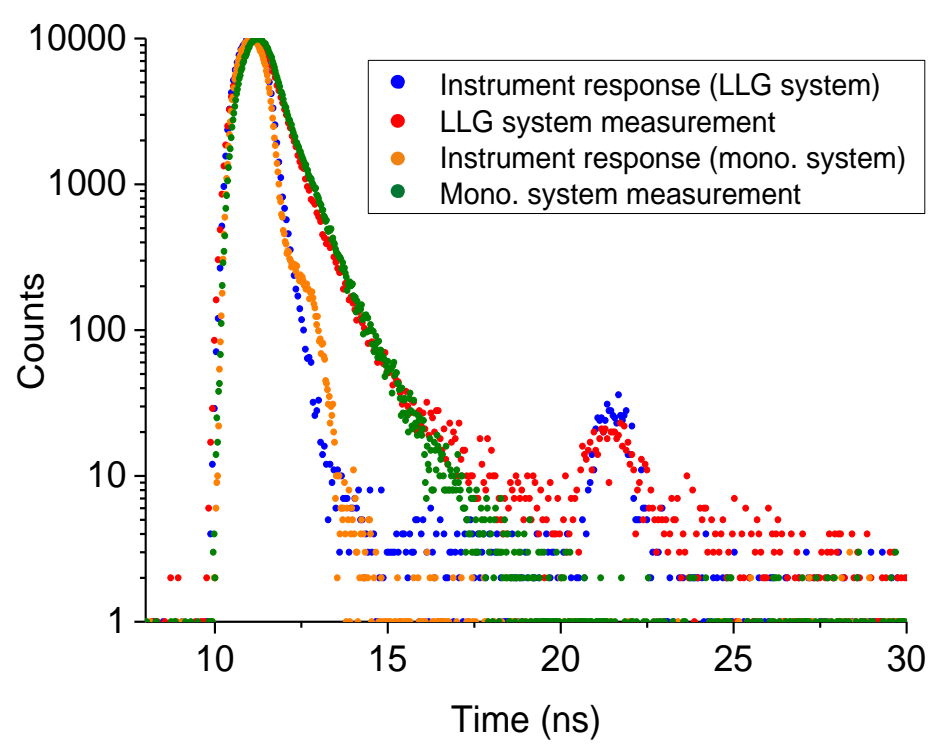

Figure 6. Fluorescence lifetime decays of NADH in Trizma on the monochromator (mono.) and liquid light guide (LLG) systems.

The fluorescent lifetime decays obtained on each system are very similar; however systematic effects that did not show up in the fluorescent dye measurements become much more prominent when working at such short timescales. Figure 6 highlights a secondary peak that occurs $\sim 10 \mathrm{~ns}$ after the initial peak in both the prompt and fluorescence lifetime measurement on the liquid light guide system. Late peaks after the instrumental pulse have previously been reported in linear focused photomultipliers ${ }^{33,34}$, however after doubling the path length by coupling two liquid light guides together ( $2 \mathrm{~m}$ length in total), a third peak $\sim 10 \mathrm{~ns}$ after the secondary peak was observed, demonstrating that it is instead a reflection occurring at the end of the liquid light guide. Despite this secondary peak occurring, there were no serious 
problems in the analysis of the fluorescence lifetime decays of $\mathrm{NADH}$, and so its appearance when measuring $\mathrm{NAD}(\mathrm{P}) \mathrm{H}$ fluorescence lifetimes in cells on this system where the fluorescence lifetime is increased will not be of concern.

\subsection{Fluorescence lifetime measurements of $\mathrm{NAD}(\mathrm{P}) \mathrm{H}$ in the cell environment}

Measurements of PNT2 cells were made on the Horiba TemPro in its original configuration as well as on the adapted liquid light guide configuration, where a comparison of the fluorescence lifetime decays as well as the corresponding lifetime components obtained for each decay are shown in Figure 7 and Table 4.

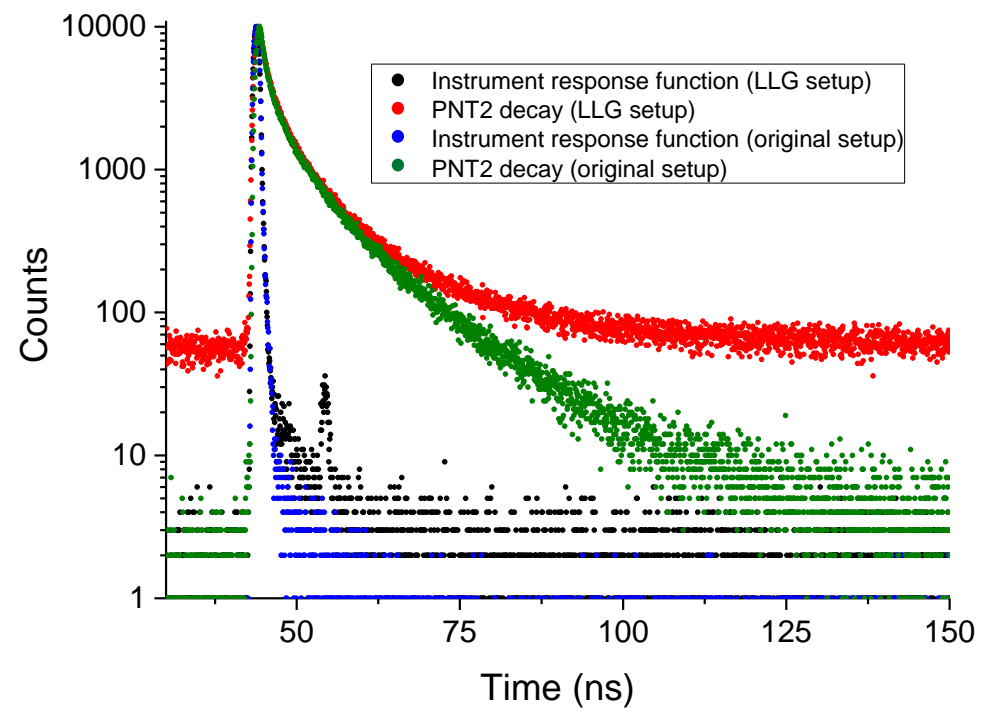

Figure 7. Fluorescence lifetime decays of PNT2 cells in suspension in PBS measured on both the original Horiba TemPro setup and the adapted liquid light guide (LLG) setup.

Table 4. Fluorescence lifetime components and corresponding contributions of each parameter from the PNT2 fluorescence lifetime decays in Figure 7.

\begin{tabular}{|c|c|c|c|c|c|c|c|}
\hline System setup & $\boldsymbol{\tau}_{\mathbf{1}}(\mathbf{n s})$ & $\mathbf{B}_{\mathbf{1}}(\boldsymbol{\%})$ & $\boldsymbol{\tau}_{\mathbf{2}}(\mathbf{n s})$ & $\mathbf{B}_{\mathbf{2}}(\boldsymbol{\%})$ & $\boldsymbol{\tau}_{\mathbf{3}}(\mathbf{n s})$ & $\mathbf{B}_{\mathbf{3}}(\boldsymbol{\%})$ & $\chi^{\mathbf{2}}$ \\
\hline Liquid light guide & $3.41 \pm 0.25$ & 43.40 & $12.0 \pm 0.3$ & 32.61 & $0.78 \pm 0.03$ & 23.99 & 1.08 \\
\hline Original & $3.73 \pm 0.21$ & 41.01 & $12.7 \pm 0.2$ & 33.13 & $0.64 \pm 0.03$ & 25.86 & 1.20 \\
\hline
\end{tabular}

Figure 7 shows that there is a significant amount of background noise in the measurement on the liquid light guide system compared to the original setup. The photon detection rate was much lower in the liquid light guide system measurement, which lead to a significantly larger acquisition time to reach 10,000 counts in the peak (890 s compared to $52 \mathrm{~s}$ ). The liquid light guide system setup is also not entirely light tight, and so the combination of this with the long acquisition time allows for a larger background noise in the measurement. Despite this background noise, Table 4 indicates that the fluorescence lifetime components obtained from this measurement are in good agreement with the measurement of the PNT2 cells on the original system setup. Looking at these components in detail, it appears that $\tau_{1}$ could correspond to $\mathrm{NAD}(\mathrm{P}) \mathrm{H}$ fluorescence. Not only does this agree with the previous statement that when in cells and bound to enzymes the NAD $(\mathrm{P}) \mathrm{H}$ lifetime increases to between $1-6.5 \mathrm{~ns}^{17}$, the three exponential fit also agrees with previous work on $\mathrm{NAD}(\mathrm{P}) \mathrm{H}$ fluorescence lifetime measurements in the cell environment ${ }^{35}$. These results demonstrate the capability of the liquid light guide system in measuring $\mathrm{NAD}(\mathrm{P}) \mathrm{H}$ fluorescence in cells, where further work in utilising its fluorescence to distinguish between high and low levels of NADPH will be carried out. 


\section{CONCLUSION}

The results presented in this paper demonstrate the potential of $\mathrm{NAD}(\mathrm{P}) \mathrm{H}$ as a fluorescent probe for tumour margin estimation, where its fluorescence lifetime has proven to be dominant in the highly scattering sol-gel environment, as well as the effect of oxygen on the fluorescence lifetime being shown as a potential mechanism for indicating which environment $\mathrm{NAD}(\mathrm{P}) \mathrm{H}$ is in. In addition to this we have demonstrated the successful use of a liquid light guide-based fluorescence lifetime system for measurements of NADH in both a simple solvent and cell environment, where a combination of using $\mathrm{NAD}(\mathrm{P}) \mathrm{H}$ as a probe and the miniaturised lifetime system pave a path towards real-time margin estimation in cancer surgery.

\section{ACKNOWLEDGEMENTS}

The authors would like to thank EPSRC and MRC for funding of the PhD studentship. Any other acknowledgements?

\section{REFERENCES}

1. World Health Organisation Media Centre (2017). Cancer Fact Sheet. Retrieved from http://www.who.int/mediacentre/factsheets/fs297/en/. Accessed 18.12.17.

2. Cancer Research UK. Cancer Statistics for the UK. Retrieved from http://www.cancerresearchuk.org/healthprofessional/cancer-statistics. Accessed 18.12.17.

3. Harmen, S., Teraphongphom, N., Tweedle, M., Basilion, J., \& Rosenthal, E. (2017). Optical Surgical Navigation for Precision in Tumor Resections. Molecular Imaging and Biology, 19(3), 357-362.

4. Mondal, S. B., Gao, S., Zhu, N., Lian, R., Gruev, V., \& Achilefu, S. (2014). Real-time Fluorescence ImageGuided Oncologic Surgery. Advances in Cancer Research, 124, 171-211.

5. Quincy Brown, J., Bydlon, T. M., Richards, L. M., Yu, B., Kennedy, S. A., Geradts, J., Wilke, L. G., Junker, M., Gallagher, J., Barry, W., \& Ramanujam, N. (2010). Optical assessment of tumor resection margins in the breast. IEEE Journal of Selected Topics in Quantum Electronics, 16(3), 530-544.

6. Boni, L., David, G., Mangano, A., Dionigi, G., Rausei, S., \& Spampatti, S. et al. (2014). Clinical applications of indocyanine green (ICG) enhanced fluorescence in laparoscopic surgery. Surgical Endoscopy, 29(7), 20462055 .

7. Keating, J., Tchou, J., Okusanya, O., Fisher, C., Batiste, R., \& Jiang, J. et al. (2016). Identification of breast cancer margins using intraoperative near-infrared imaging. Journal of Surgical Oncology, 113(5), 508-514.

8. Liu, J., Guo, W., \& Tong, M. (2016). Intraoperative indocyanine green fluorescence guidance for excision of nonpalpable breast cancer. World Journal Of Surgical Oncology, 14(1).

9. Owens, E. A., Henary, M., Fakhri, G. E., \& Choi, H. S. (2016). Tissue-Specific Near-Infrared Fluorescence Imaging. Accounts of Chemical Research, 49(9), 1731-1740.

10. Khan Academy. Cellular Respiration: Introduction to cellular respiration and redox. Retrieved from: https://www.khanacademy.org/science/biology/cellular-respiration-and-fermentation/intro-to-cellularrespiration/a/intro-to-cellular-respiration-and-redox. Accessed 18.12.17.

11. Patra, K. C., Hay, N. (2014). The pentose phosphate pathway and cancer. Trends in Biochemical Sciences, 39(8), 347-354.

12. Cairns, R., Harris, I., \& Mak, T. (2011). Regulation of cancer cell metabolism. Nature Reviews Cancer, 11, 8595.

13. Alberghina, L., \& Gaglio, D. (2014). Redox control of glutamine utilization in cancer. Cell Death and Disease, 5(12), e1561.

14. Blacker, T. S, \& Duchen, M. R. (2016). Investigating mitochondrial redox state using NADH and NADPH autofluorescence. Free Radical Biology and Medicine, 100, 53-65. 
15. Heikal, A. A. (2010). Intracellular coenzymes as natural biomarkers for metabolic activities and mitochondrial anomalies. Biomarkers in Medicine, 4(2), 241-263.

16. Blacker, T. S., Mann, Z. F., Gale, J. E, Ziegler, M., Bain, A. J., Szabadkai, G., \& Duchen, M. R. (2014). Separating NADH and NADPH fluorescence in live cells and tissues using FLIM. Nature Communications, 5, 3936.

17. Blacker, T. S, Mann, Z. F., Gale, J. E., Ziegler, M., Bain, A. J., Szabadkai, G., \& Duchen, M. R. (2014). Separating NADH and NADPH fluorescence in live cells and tissues using FLIM. Nature Communications, 5, $1-9$.

18. Butte, P. V., Mamelak, A. N., Nuno, M., Bannykh, S. I., Black, K. L., \& Marcu, L. (2011). Fluorescence lifetime spectroscopy for guided therapy of brain tumors. Neuroimage, 54, S125-S135.

19. Birch D. J. S., \& Geddes C. D. (2000). Sol-gel particle growth studied using fluorescence anisotropy: an alternative to scattering techniques. Physical Review E, 62, 2977-80.

20. Stewart, H. L, Yip, P., Rosenberg, M., Sorensen, T. J., Laursen B. W., Knight, A. E., \& D. J. S. Birch (2016). Nanoparticle metrology of silica colloids and super-resolution studies using the ADOTA fluorophore. Measurement Science and Technology, 274(4), 045007.

21. Hungerford, G., Rei, A., Ferreira, M. I. C., Suhling, K., \& Tregidgo, C. (2007). Diffusion in a Sol-Gel-Derived Medium with a View toward Biosensor Applications. The Journal of Physical Chemistry B, 111(13), 35583562.

22. Selanger, K. A., Falnes, J., \& Sikkeland, T. (1977). Fluorescence lifetime studies of Rhodamine 6G in methanol. The Journal Of Physical Chemistry, 81(20), 1960-1963.

23. Geddes, C. D., \& Birch, D. J. S. (2000). Nanometre resolution of silica hydrogel formation using time-resolved fluorescence anisotropy. Journal Of Non-Crystalline Solids, 270(1-3), 191-204.

24. Birch, D. J. S., \& Yip, P. (2014). Nanometrology. In Fluorescence Spectroscopy and Microscopy: Methods and Protocols (pp. 279-301). New York, NY: Hamana Press.

25. Lumatec (2017). Lumatec Liquid Light Guides. Retrieved from: http://www.lumatec.de/en/liquid-lightguide/. Accessed 15.05.17.

26. Sanderson, A., Holmes, A. S., Birch, D. J. S., \& Imhof, R. E. (1993). A single-photon timing fluorometer with liquid light guide sensing. SPIE Proceedings, 1885, 466-477.

27. Birch, D. J. S., Sanderson, A., Holmes, A. S., McLoskey, D., \& Imhof, R. E. (1993). Miniaturised single-photon fluorometry. Measurement Science and Technology, 4, 797-799.

28. Sircus, M (March 2010). Oxygen and Cancer. Retrieved from http://drsircus.com/cancer/oxygen-and-cancer/. Accessed 19.12.17.

29. Cooper, G. \& Hausman, R. (2000). The Cell: A Molecular Approach (2nd edition). Sunderland, MA: Sinauer Associates.

30. Wagnieres, G., Cheng, S., Zellweger, M., Utke, N., Braichotte, D., Ballini, J., \& Bergh, H. (1996). An optical phantom with tissue-like properties in the visible for use in PDT and fluorescence spectroscopy. Physics in Medicine \& Biology, 42, 1415-1426.

31. De Grand, A. M., Lomnes, S. J., Lee, D. S., Pietrzykowski, M., Ohnishi, S., Morgan, T. G., Gogbashian, A., Laurence, R. G., \& Frangioni, J. V. (2006). Tissue-like Phantoms for Near-Infrared Fluorescence Imagine System Assessment and the Training of Surgeons. Journal of Biomedical Optics, 11(1): 014007.

32. Bude, R. O., \& Adler, R. S. (1995). An Easily Made, Low-Cost, Tissue-Like Ultrasound Phantom Material. Journal of Clinical Ultrasound, 23, 271-273.

33. Birch, D. J. S., Hungerford, G., Nadolski, B., Imhof, R. E., \& Dutch, A. D. (1988). Time-correlated singlephoton counting fluorescence decay studies at $930 \mathrm{~nm}$ using spark source excitation. Journal Of Physics E: Scientific Instruments, 21(9), 857-862.

34. Birch, D. J. S., Imhof, R. E., and Dutch, A. D. (1984). Pulse fluorometry using simultaneous acquisition of fluorescence and excitation. Review of Scientific Instruments, 55(8), 1255-1644. 
35. Evans, N. D., Gnudi, L., Rolinski, O. J., Birch, D. J. S., \& Pickup, J. C. (2005). Glucose-dependent changes in NAD(P)H-related fluorescence lifetime of adipocytes and fibroblasts in vitro: Potential for non-invasive glucose sensing in diabetes mellitus. Journal of Photochemistry and Photobiology B: Biology, 80, 122-129. 